\title{
A Class of Fractional $p$-Laplacian Integrodifferential Equations in Banach Spaces
}

\author{
Yiliang Liu' ${ }^{1}$ and Liang $\mathrm{Lu}^{1,2}$ \\ ${ }^{1}$ Guangxi Key Laboratory of Hybrid Computation and IC Design Analysis and College of Sciences, Guangxi University for Nationalities, \\ Nanning, Guangxi 530006, China \\ ${ }^{2}$ College of Sciences, Hezhou University, Hezhou, Guangxi 542899, China
}

Correspondence should be addressed to Yiliang Liu; yiliangliu100@126.com

Received 17 April 2013; Accepted 26 June 2013

Academic Editor: Paul Eloe

Copyright (C) 2013 Y. Liu and L. Lu. This is an open access article distributed under the Creative Commons Attribution License, which permits unrestricted use, distribution, and reproduction in any medium, provided the original work is properly cited.

We study a class of nonlinear fractional integrodifferential equations with $p$-Laplacian operator in Banach space. Some new existence results are obtained via fixed point theorems for nonlocal boundary value problems of fractional $p$-Laplacian equations. An illustrative example is also discussed.

\section{Introduction}

In this paper, we discuss a class of fractional integrodifferential equations with $p$-Laplacian operator and nonlocal boundary condition in Banach space $E$ :

$$
\begin{gathered}
D_{0^{+}}^{\beta} \phi_{p}\left(D_{0^{+}}^{\alpha} x(t)+g(t, x(t))\right)=f(t, x(t), S x(t)), \\
t \in[0, T], \\
x(0)+x(T)=D_{0^{+}}^{\alpha} x(0)+D_{0^{+}}^{\alpha} x(T)=\theta, \\
\eta_{1} x^{\prime}(0)+\eta_{2} x^{\prime}(T)=\bar{g}(x),
\end{gathered}
$$

where $1<\alpha \leq 2,0<\beta \leq 1,2<\alpha+\beta \leq 3, D_{0^{+}}^{\alpha}$ denotes the Caputo fractional derivative of order $\alpha . \phi_{p}(x)=\|x\|^{p-2} x(p>$ 1 ) is called a $p$-Laplacian operator. $T>0$ is a constant, $\theta$ is the zero element of $E$, and $\eta_{1}, \eta_{2} \in \mathbb{R}, f \in C\left([0, T] \times E^{2}, E\right), h, g \in$ $C([0, T] \times E, E), S x(t)=\int_{0}^{t} h(s, x(s)) d s$, and $\bar{g}: C(0, T ; E) \rightarrow$ $E$ is a continuous functional.

Recently, fractional differential equations with $p$-Laplacian operator have been widely applied in many fields of physics and natural phenomena, such as non-Newtonian mechanics, fluid mechanics, viscoelasticity mechanics, combustion theory, and material science. There have appeared some results for the existence of solutions of BVPs for fractional differential equations with $p$-Laplacian operator, see [1-11] and the references therein.

In the last few years, the research of antiperiodic BVPs has received considerable attention and become a much important area. The study of antiperiodic solutions for nonlinear evolution equations is closely related to the study of periodic solutions, and it was initiated by Okochi [12]. And antiperiodic boundary conditions appear in physics in a variety of situations (cf. [13-15] and references therein).

As well as we know, it has been shown, first by Tavazoei et al. and later by Kaslik and Sivasundaram (cf. [16-19]), that periodic solution in fractional dynamical systems does not exist. Therefore antiperiodic solutions may not exist for fractional differential equations. However, such kind of boundary condition (the value at endpoint has different signs) still received considerable attention; it may involve some resonance problems. For example, Chen and Liu [3] studied a kind of BVP for the fractional $p$-Laplacian equation as follows:

$$
\begin{gathered}
{ }^{C} D_{0^{+}}^{\beta} \phi_{p}\left({ }^{C} D_{0^{+}}^{\alpha} u(t)\right)=f(t, u(t)), \quad t \in[0,1], \\
u(0)=-u(1), \quad D_{0^{+}}^{\alpha} u(0)=-D_{0^{+}}^{\alpha} u(1),
\end{gathered}
$$

where $0<\alpha, \beta \leq 1,1<\alpha+\beta \leq 2,{ }^{C} D_{0^{+}}^{\alpha}$ is a standard Caputo fractional derivative and $f:[0,1] \times \mathbb{R} \rightarrow \mathbb{R}$ is continuous. Under certain nonlinear growth conditions of 
the nonlinearity, the existence result was obtained by using Schaefer's fixed point theorem.

Alsaedi [13] proved some existence results for a BVP for fractional differential equations as mentioned later:

$$
\begin{gathered}
{ }^{C} D_{0^{+}}^{q} x(t)=f(t, x(t),(\chi x)(t)), \quad t \in[0, T], \\
x(0)=-x(T), \quad x^{\prime}(0)=-x^{\prime}(T),
\end{gathered}
$$

where ${ }^{C} D_{0^{+}}^{q}$ denotes the standard Caputo fractional derivative of order $1<q \leq 2, f:[0, T] \times E^{2} \rightarrow E$, and for $\gamma:$ $[0, T] \times[0, T] \rightarrow[0, \infty),(\chi x)(t)=\int_{0}^{t} \gamma(t, s) x(s) d s,(E,\|\cdot\|)$ is a Banach space. The contraction mapping principle and Krasnoselskii's fixed point theorem are applied to establish the results in [13].

Moreover, if $g(t, x(t))=\lambda x(t), \lambda \in \mathbb{R}, p=2$, then (1) reduces to the Langevin equation which has been widely used to describe the evolution of physical phenomena in fluctuating environments. In [20], the authors studied such type of Langevin equation with two different fractional orders. This new version of fractional Langevin equation gives a fractional Gaussian process parameterized by two indices, which provides a more flexible model for fractal processes as compared with the usual one characterized by a single index. In [21], the fractional oscillator process with two indices was discussed. For more details, see [22-26] and references therein.

In [24], Ahmad et al. discussed the existence solutions for the three-point BVPs of Langevin equation with two different fractional orders:

$$
\begin{gathered}
{ }^{C} D_{0^{+}}^{\beta}\left({ }^{C} D_{0^{+}}^{\alpha}+\alpha+\lambda\right) x(t)=f(t, x(t)), \quad t \in(0,1), \\
x(0)=0, \quad x(\eta)=0, \quad x(1)=0, \quad 0<\eta<1,
\end{gathered}
$$

where ${ }^{C} D_{0^{+}}^{\alpha}$ denotes the standard Caputo fractional derivative, $0<\alpha \leq 1,1<\beta \leq 2, f \in C([0,1] \times \mathbb{R}, \mathbb{R})$, and $\lambda$ is a real number. Ahmad and Nieto [25] studied a Dirichlet BVP of Langevin equation

$$
\begin{array}{r}
{ }^{C} D_{0^{+}}^{\beta}\left({ }^{C} D_{0^{+}}^{\alpha}+\lambda\right) x(t)=f(t, x(t)), \\
t \in(0,1), \quad 0<\alpha, \quad \beta \leq 1, \\
x(0)=\gamma_{1}, \quad x(1)=\gamma_{2},
\end{array}
$$

where $f:[0,1] \times E \rightarrow E, \lambda$ is a real number, $\gamma_{1}, \gamma_{2} \in E$. Here, $(E,\|\cdot\|)$ is a Banach space. A. P. Chen and Y. Chen [26] considered the BVP of Langevin equation with two different fractional orders:

$$
\begin{gathered}
{ }^{C} D_{0^{+}}^{\beta}\left({ }^{C} D_{0^{+}}^{\alpha}+\lambda\right) u(t)=f(t, u(t)), \\
t \in(0, T), \quad T>0, \\
u(0)=-u(T), \quad u^{\prime}(0)=u^{\prime}(T)=0,
\end{gathered}
$$

where $1<\alpha \leq 2,0<\beta \leq 1, f:[0, T] \times \mathbb{R} \rightarrow \mathbb{R}$ is continuous and $\lambda$ is a real number. By applying contraction mapping principle and Krasnoselskii's fixed point theorem, some existence results are obtained in [24-26].

Motivated by previously mentioned works, we will consider the existence of solutions of fractional $p$-Laplacian BVP (1) with nonlocal boundary condition. To authors' knowledge, there are few results on the existence of solutions of nonlinear fractional $p$-Laplacian differential equations in Banach spaces, and no paper is concerned with the existence results for fractional $p$-Laplacian integrodifferential equation (1). And the main difficulty that, for $p \neq 2$, it is impossible for us to find a Green's function in the equivalent integral operator since the differential operator $D_{0^{+}}^{\beta} \phi_{p}\left(D_{0^{+}}^{\alpha}\right)$ is nonlinear. This paper is concerned with BVP (1) by using some known fixed point theorems. Such investigations will provide an important platform for gaining a deeper understanding of nature.

The paper is organized as follows. In Section 2, we present some material. In Section 3, by applying Krasnoselskii's theorem and Schauder's fixed point theorem, the existence of solutions is given for nonlinear fractional BVP (1). Finally, an example is shown in Section 4 to illustrate the usefulness of the main results.

\section{Preliminaries and Lemmas}

Firstly, we recall the following known definitions, which can be found in $[4,10]$.

Definition 1. The Riemann-Liouville fractional integral operator of order $\gamma>0$ of a function $f:(0, \infty) \rightarrow \mathbb{R}$ is given by

$$
I_{0^{+}}^{\gamma} f(t)=\frac{1}{\Gamma(\gamma)} \int_{0}^{t}(t-s)^{\gamma-1} f(s) d s,
$$

provided that the right side integral is pointwise defined on $(0,+\infty)$.

Definition 2. The Riemann-Liouville derivative of order $\gamma>0$ for a function $f:[0, \infty) \rightarrow \mathbb{R}$ can be written as

$$
{ }^{L} \mathbf{D}_{0^{+}}^{\gamma} f(t)=\frac{1}{\Gamma(n-\gamma)} \frac{d^{n}}{d t^{n}} \int_{0}^{t} \frac{f(s)}{(t-s)^{\gamma-n+1}} d s,
$$

where $n$ is the smallest integer greater than $\gamma$.

Definition 3. The Caputo fractional derivative of order $\gamma>0$ for a function $f:[0, \infty) \rightarrow \mathbb{R}$ can be written as

$$
D_{0^{+}}^{\gamma} f(t)={ }^{L} \mathbf{D}_{0^{+}}^{\gamma}\left(f(t)-\sum_{k=0}^{n-1} \frac{t^{k}}{k !} f^{(k)}(0)\right),
$$

where $n$ is the smallest integer greater than $\gamma$.

For $n \in \mathbb{N}^{+}:=\{1,2, \ldots\}, A C^{n}[a, b]$ denotes the space of functions $f(t)$ which have continuous derivatives up to order $n-1$ on $[a, b]$ such that $f^{(n-1)}(t) \in A C[a, b]$ :

$$
A C^{n}[a, b]=\left\{f:[a, b] \longrightarrow \mathbb{R}, f^{(n-1)}(t) \in A C[a, b]\right\},
$$

where $A C[a, b]$ is the space of absolutely continuous functions on $[a, b]$. Then we easily get the following. 
Remark 4. If $u \in A C^{n}[0, \infty)$, then it is the standard Caputo fractional derivative

$$
D_{0^{+}}^{\gamma} u(t)={ }^{C} D_{0^{+}}^{\gamma} u(t):=\frac{1}{\Gamma(n-\gamma)} \int_{0}^{t} \frac{u^{(n)}(s)}{(t-s)^{\gamma+1-n}} d s,
$$

where $n$ is the smallest integer greater than $\gamma$. Furthermore, the Caputo derivative of a constant is equal to zero.

Lemma 5 (see [4]). Let $\gamma>0$. Assume that $u \in A C^{n}[0, \infty)$. Then the following equality holds:

$$
I_{0^{+}}^{\gamma} D_{0^{+}}^{\gamma} u(t)=I_{0^{+}}^{\gamma}{ }^{C} D_{0^{+}}^{\gamma} u(t)=u(t)+c_{0}+c_{1} t+\cdots+c_{n-1} t^{n-1},
$$

where $c_{i} \in \mathbb{R}, i=0,1, \ldots, n-1$; here $n$ is the smallest integer greater than $\gamma$.

The following famous fixed point theorems will be used to prove the existence results of BVP (1).

Lemma 6 (Krasnoselskii's Theorem [27]). Let $M$ be a closed convex and nonempty subset of a Banach space X. Let $A, B$ be the operators such that

(i) $A x+B y \in M$ whenever $x, y \in M$;

(ii) $A$ is compact and continuous;

(iii) $B$ is a contraction mapping.

Then there exists $z \in M$ such that $z=A z+B z$.

Lemma 7 (Schauder's fixed point theorem). If $\Omega$ is a nonempty closed bounded convex subset of a Banach space $X$, and $T: \Omega \rightarrow \Omega$ is completely continuous, then $T$ has a fixed point in $\Omega$.

\section{Existence Results}

In this section, we deal with the existence of solutions of the BVP (1).

At first, we introduce the Banach space $X=C(0, T ; E)$ of all continuous functions from $[0, T]$ to $E$ endowed with a topology of uniform convergence with norm defined by $\|x\|_{X}=\sup \{\|x(t)\|: t \in[0, T]\}$, and $\|\cdot\|$ denotes the norm in Banach space $E$.

In relation to (1), we introduce the following fractional $p$ Laplacian differential equations:

$$
\begin{gathered}
D_{0^{+}}^{\beta} \phi_{p}\left(D_{0^{+}}^{\alpha} x(t)+g(t, x(t))\right)=h(t), \quad t \in[0, T], \\
x(0)+x(T)=\theta, \quad D_{0^{+}}^{\alpha} x(0)+D_{0^{+}}^{\alpha} x(T)=\theta, \\
\eta_{1} x^{\prime}(0)+\eta_{2} x^{\prime}(T)=x_{0},
\end{gathered}
$$

where $\eta_{1}, \eta_{2} \in \mathbb{R}, x_{0} \in E$.

Remark 8. Obviously, $\phi_{p}$ is invertible and $\left(\phi_{p}\right)^{-1}=\phi_{q}$, where $q>1$ such that $1 / p+1 / q=1 . \phi_{p}$ and $\phi_{q}$ are strictly increasing functions. Furthermore, the nonlinear operator $D_{0^{+}}^{\beta} \phi_{p}\left(D_{0^{+}}^{\alpha}\right)$ reduces to the linear operator $D_{0^{+}}^{\beta} D_{0^{+}}^{\alpha}$ when $p=2$, and the additive index law $D_{0^{+}}^{\beta} D_{0^{+}}^{\alpha} x(t)=D_{0^{+}}^{\beta+\alpha} x(t)$ holds under reasonable conditions on the function $x(t)$ (see $[4,10])$.

Lemma 9. Let $\omega \in X$ and $\eta_{1}+\eta_{2} \neq 0$; then the unique solution of the BVP,

$$
\begin{aligned}
& D_{0^{+}}^{\alpha} x(t)=\omega(t), \quad t \in[0, T], T>0,1<\alpha \leq 2, \\
& x(0)+x(T)=\theta, \quad \eta_{1} x^{\prime}(0)+\eta_{2} x^{\prime}(T)=x_{0},
\end{aligned}
$$

is given by

$$
x(t)=\int_{0}^{T} G(t, s) \omega(s) d s+\frac{(2 t-T) x_{0}}{2\left(\eta_{1}+\eta_{2}\right)}, \quad t \in[0, T],
$$

where $G(t, s)$ is given by

$G(t, s)$

$$
= \begin{cases}\frac{2(t-s)^{\alpha-1}-(T-s)^{\alpha-1}}{2 \Gamma(\alpha)}+\frac{\eta_{2}(T-t)(T-s)^{\alpha-2}}{2\left(\eta_{1}+\eta_{2}\right) \Gamma(\alpha-1)}, & s \leq t, \\ \frac{\eta_{2}(T-t)(T-s)^{\alpha-2}}{2\left(\eta_{1}+\eta_{2}\right) \Gamma(\alpha-1)}-\frac{(T-s)^{\alpha-1}}{2 \Gamma(\alpha)}, & t \leq s .\end{cases}
$$

Proof. Assume that $x(t)$ satisfies the equation of (14); then by the first equality of (14) and Lemma 5, we have

$$
\begin{gathered}
x(t)=c_{0}+c_{1} t+\int_{0}^{t} \frac{(t-s)^{\alpha-1}}{\Gamma(\alpha)} \omega(s) d s \\
x^{\prime}(t)=c_{1}+\int_{0}^{t} \frac{(t-s)^{\alpha-2}}{\Gamma(\alpha-1)} \omega(s) d s
\end{gathered}
$$

Applying the boundary conditions of (14), thus

$$
\begin{aligned}
& c_{0}+\left(c_{0}+c_{1} T+\int_{0}^{T} \frac{(T-s)^{\alpha-1}}{\Gamma(\alpha)} \omega(s) d s\right)=\theta, \\
& \eta_{1} c_{1}+\eta_{2}\left(c_{1}+\int_{0}^{T} \frac{(T-s)^{\alpha-2}}{\Gamma(\alpha-1)} \omega(s) d s\right)=x_{0} .
\end{aligned}
$$

Through some calculation, we get

$$
\begin{aligned}
c_{1}= & -\int_{0}^{T} \frac{\eta_{2}(T-s)^{\alpha-2}}{\left(\eta_{1}+\eta_{2}\right) \Gamma(\alpha-1)} \omega(s) d s+\frac{x_{0}}{\eta_{1}+\eta_{2}}, \\
c_{0}= & -\frac{T}{2} c_{1}-\int_{0}^{T} \frac{(T-s)^{\alpha-1}}{2 \Gamma(\alpha)} \omega(s) d s \\
= & \int_{0}^{T} \frac{T \eta_{2}(T-s)^{\alpha-2}}{2\left(\eta_{1}+\eta_{2}\right) \Gamma(\alpha-1)} \omega(s) d s \\
& -\int_{0}^{T} \frac{(T-s)^{\alpha-1}}{2 \Gamma(\alpha)} \omega(s) d s-\frac{x_{0} T}{2\left(\eta_{1}+\eta_{2}\right)} .
\end{aligned}
$$


Thus

$$
\begin{aligned}
x(t)= & \int_{0}^{T} \frac{T \eta_{2}(T-s)^{\alpha-2}}{2\left(\eta_{1}+\eta_{2}\right) \Gamma(\alpha-1)} \omega(s) d s \\
& -\int_{0}^{T} \frac{(T-s)^{\alpha-1}}{2 \Gamma(\alpha)} \omega(s) d s-\frac{x_{0} T}{2\left(\eta_{1}+\eta_{2}\right)} \\
& -t \int_{0}^{T} \frac{\eta_{2}(T-s)^{\alpha-2}}{\left(\eta_{1}+\eta_{2}\right) \Gamma(\alpha-1)} \omega(s) d s+\frac{x_{0} t}{\eta_{1}+\eta_{2}} \\
& +\int_{0}^{t} \frac{(t-s)^{\alpha-1}}{\Gamma(\alpha)} \omega(s) d s \\
= & \int_{0}^{T} G(t, s) \omega(s) d s+\frac{(2 t-T) x_{0}}{2\left(\eta_{1}+\eta_{2}\right)}, \quad t \in[0, T] .
\end{aligned}
$$

This completes the proof.

Lemma 10. The function $G(t, s)$ satisfy

$$
\begin{aligned}
\left|\int_{0}^{T} G(t, s) d s\right| & \leq \int_{0}^{T}|G(t, s)| d s \\
& \leq \frac{\left|\eta_{2}\right| T^{\alpha}}{2\left|\eta_{1}+\eta_{2}\right| \Gamma(\alpha)}+\frac{3 T^{\alpha}}{2 \Gamma(\alpha+1)} \\
& :=\mu_{0}, \quad t \in[0, T] .
\end{aligned}
$$

Proof. It is easy to see that, for $t \in[0, T]$, we obtain

$$
\begin{aligned}
\left|\int_{0}^{T} G(t, s) d s\right| \leq & \int_{0}^{T}|G(t, s)| d s \\
\leq & \int_{0}^{T} \frac{\eta_{2}(T-t)(T-s)^{\alpha-2}}{2\left|\eta_{1}+\eta_{2}\right| \Gamma(\alpha-1)} d s \\
& +\int_{0}^{T} \frac{(T-s)^{\alpha-1}}{2 \Gamma(\alpha)} d s+\int_{0}^{t} \frac{(t-s)^{\alpha-1}}{\Gamma(\alpha)} d s \\
\leq & \frac{\left|\eta_{2}\right|(T-t) T^{\alpha-1}}{2\left|\eta_{1}+\eta_{2}\right| \Gamma(\alpha)}+\frac{T^{\alpha}}{2 \Gamma(\alpha+1)}+\frac{t^{\alpha}}{\Gamma(\alpha+1)} \\
\leq & \frac{\left|\eta_{2}\right| T^{\alpha}}{2\left|\eta_{1}+\eta_{2}\right| \Gamma(\alpha)}+\frac{3 T^{\alpha}}{2 \Gamma(\alpha+1)}:=\mu_{0} .
\end{aligned}
$$

This completes the proof.

Lemma 11. Assume that $h \in X$ and $g \in C([0, T] \times E, E)$ satisfies $g(0,-u)=-g(T, u)$ for any $u \in E$; then $x \in\{x$ : $x(\cdot) \in A C(0, T ; E)$ and $\left.\phi_{p}\left(D_{0^{+}}^{\alpha} x(\cdot)+g(\cdot, x(\cdot))\right) \in A C(0, T ; E)\right\}$ is a solution of (13) if and only if $x \in C(0, T ; E)$ is a solution of the following integral equation:

$$
\begin{aligned}
x(t)= & \int_{0}^{T} G(t, s)\left[\phi_{q}(F h(s))-g(s, x(s))\right] d s \\
& +\frac{(2 t-T) x_{0}}{2\left(\eta_{1}+\eta_{2}\right)}, \quad t \in[0, T],
\end{aligned}
$$

where $G(t, s)$ is given by (16) and

$$
\begin{array}{r}
F h(t)=\int_{0}^{t} \frac{(t-s)^{\beta-1}}{\Gamma(\beta)} h(s) d s-\int_{0}^{T} \frac{(T-s)^{\beta-1}}{2 \Gamma(\beta)} h(s) d s, \\
t \in[0, T] .
\end{array}
$$

Proof. Assume that $x$ is a solution of (13). Then by (13) and Lemma 5, we have

$$
\begin{aligned}
\phi_{p}\left(D_{0^{+}}^{\alpha} x(t)\right. & +g(t, x(t))) \\
& =c_{0}+\int_{0}^{t} \frac{(t-s)^{\beta-1}}{\Gamma(\beta)} h(s) d s, \quad t \in[0, T] .
\end{aligned}
$$

For $\phi_{p}^{-1}=\phi_{q}$, we can get

$$
\begin{array}{r}
D_{0^{+}}^{\alpha} x(t)=-g(t, x(t))+\phi_{q}\left(c_{0}+\int_{0}^{t} \frac{(t-s)^{\beta-1}}{\Gamma(\beta)} h(s) d s\right), \\
t \in[0, T] .
\end{array}
$$

Combining with $D_{0^{+}}^{\alpha} x(0)=-D_{0^{+}}^{\alpha} x(T)$, we obtain

$$
\begin{aligned}
& -g(0, x(0))+\phi_{q}\left(c_{0}\right) \\
& \quad=g(T, x(T))-\phi_{q}\left(c_{0}+\int_{0}^{T} \frac{(T-s)^{\beta-1}}{\Gamma(\beta)} h(s) d s\right) .
\end{aligned}
$$

Since $x(0)=-x(T), g(T, x(T))=-g(0,-x(T))=-g(0$, $x(0))$ and $\phi_{q}(-s)=-\phi_{q}(s)$, thus $(27)$ yields that

$$
c_{0}=-\frac{1}{2} \int_{0}^{T} \frac{(T-s)^{\beta-1}}{\Gamma(\beta)} h(s) d s .
$$

Substituting previous equation into (25), then

$$
\begin{aligned}
\phi_{p}\left(D_{0^{+}}^{\alpha} x(t)+g(t, x(t))\right) \\
\quad=\int_{0}^{t} \frac{(t-s)^{\beta-1}}{\Gamma(\beta)} h(s) d s-\frac{1}{2} \int_{0}^{T} \frac{(T-s)^{\beta-1}}{\Gamma(\beta)} h(s) d s \\
=: F h(t), \quad t \in[0, T],
\end{aligned}
$$

which means that

$$
D_{0^{+}}^{\alpha} x(t)=\phi_{q}(F h(t))-g(t, x(t)), \quad t \in[0, T] .
$$

Now, setting $\omega(t)=\phi_{q}(F h(t))-g(t, x(t))$, then (13) can be changed into the form of (14). Applying Lemma 9, we can get (23).

Conversely, we can obtain that the solution of (23) is the solution of the BVP (13) by calculation, which completes our proof.

Now, let us consider the existence of solutions of BVP for $p$-Laplacian equations (1). Let $N$ be a Nemytskii operator defined by

$$
N x(t)=f(t, x(t), S x(t)), \quad t \in[0, T],
$$


and for $t \in[0, T]$, we denote

$$
F N x(t)=\int_{0}^{t} \frac{(t-s)^{\beta-1}}{\Gamma(\beta)} N x(s) d s-\int_{0}^{T} \frac{(T-s)^{\beta-1}}{2 \Gamma(\beta)} N x(s) d s .
$$

We define an operator $\mathscr{F}: X \rightarrow X$ as follows:

$$
\begin{aligned}
\mathscr{F} x(t)= & \int_{0}^{T} G(t, s)\left[\phi_{q}(F N x(s))-g(s, x(s))\right] d s \\
& +\frac{(2 t-T)}{2\left(\eta_{1}+\eta_{2}\right)} \bar{g}(x), \quad t \in[0, T],
\end{aligned}
$$

where $G(t, s)$ is given by (16). Clearly, a fixed point of the operator $\mathscr{F}$ is a solution of the problem (1).

In the sequel, we need the following assumptions.

$\left(\mathrm{H}_{1}\right)$ Let $f:[0, T] \times E^{2} \rightarrow E$ be continuous and $\exists a, b, c \in$ $\mathbb{R}^{+}$such that

$$
\begin{array}{r}
\|f(t, u, v)\| \leq a+b\|u\|^{p-1}+c\|v\|^{p-1} \\
\forall t \in[0, T], \quad u, v \in E .
\end{array}
$$

$\left(\mathrm{H}_{2}\right)$ The function $g:[0, T] \times E \rightarrow E$ is continuous and $g(0,-u)=-g(T, u)$ for all $u \in E$. Moreover, $\exists L>0$ such that

$$
\begin{array}{r}
\|g(t, u)-g(t, v)\| \leq L\|u-v\| \\
\forall t \in[0, T], \quad u, v \in E .
\end{array}
$$

$\left(\mathrm{H}_{3}\right)$ Let $h \in([0, T] \times E, E)$, and $\exists k \in L^{1}(0, T ; \mathbb{R})$ satisfies

$$
\|h(t, u)\| \leq k(t)\|u\| \quad \forall t \in[0, T], u \in E .
$$

$\left(\mathrm{H}_{4}\right)$ Let $\bar{g}: C(0, T ; E) \rightarrow E$ be a continuous functional and satisfies

$$
\left\|\bar{g}\left(x_{1}\right)-\bar{g}\left(x_{2}\right)\right\| \leq M\left\|x_{1}-x_{2}\right\|_{X} \quad \forall x_{1}, x_{2} \in X
$$

Theorem 12. Assume that $\left(H_{1}\right)-\left(H_{4}\right)$ hold. If

$$
\rho=\mu_{0}\left(\frac{9 T^{\beta}\left(b+c\|k\|_{L^{1}}^{p-1}\right)}{4 \Gamma(\beta+1)}\right)^{q-1}+\frac{5}{4} \mu_{0} L+\frac{4 T M+1}{8\left|\eta_{1}+\eta_{2}\right|}<1,
$$

where $\mu_{0}$ is defined in Lemma 10, Then BVP (1) has at least one solution in $X$.

Proof. Let us define a bounded set $B_{r}=\{x: x \in X$ and $\left.\|x\|_{X} \leq r\right\}$, where

$$
r \geq \max \left\{\frac{4 g_{0}}{L},\left(\frac{2 a}{b+c\|k\|_{L^{1}}^{p-1}}\right)^{q-1}, 4 T\|\bar{g}(\theta)\|\right\},
$$

and $g_{0}=\sup _{t \in[0, T]}\|g(t, \theta)\|$. Then $B_{r}$ is a closed convex and nonempty subset of a Banach space $X$. We define two operators $A$ and $B$ on $B_{r} \subset X$ such that $\mathscr{F}=A+B$ and

$$
\begin{aligned}
& A x(t)=\int_{0}^{T} G(t, s) \phi_{q}(F N x(s)) d s, \\
& B x(t)=-\int_{0}^{T} G(t, s) g(s, x(s)) d s+\frac{(2 t-T)}{2\left(\eta_{1}+\eta_{2}\right)} \bar{g}(x) .
\end{aligned}
$$

Firstly, we show that $A x+B y \in B_{r}$ for any $x, y \in B_{r}$. From the assumptions $\left(\mathrm{H}_{1}\right)$ and $\left(\mathrm{H}_{3}\right)$, we have

$$
\begin{aligned}
\|S x(t)\| \leq & \int_{0}^{t}\|h(s, x(s))\| d s \\
\leq & \int_{0}^{T} k(s)\|x(s)\| d s \\
\leq & \|k\|_{L^{1}}\|x\|_{X}\left(\operatorname{see}\left(\mathrm{H}_{3}\right)\right) \\
\Longrightarrow & \|N x(t)\|=\|f(t, x(t), S x(t))\| \\
\leq & a+b\|x(t)\|^{p-1}+c\|S x(t)\|^{p-1}\left(\operatorname{see}\left(\mathrm{H}_{1}\right)\right) \\
\leq & a+b\|x\|_{X}^{p-1}+c\left(\|k\|_{L^{1}}\|x\|_{X}\right)^{p-1} \\
\Longrightarrow & \|F N x(t)\| \\
\leq & \int_{0}^{t} \frac{(t-s)^{\beta-1}}{\Gamma(\beta)}\|N x(s)\| d s \\
& +\int_{0}^{T} \frac{(T-s)^{\beta-1}}{2 \Gamma(\beta)}\|N x(s)\| d s \\
\leq & \left(\int_{0}^{t} \frac{(t-s)^{\beta-1}}{\Gamma(\beta)} d s+\int_{0}^{T} \frac{(T-s)^{\beta-1}}{2 \Gamma(\beta)} d s\right) \\
& \times\left[a+b\|x\|_{X}^{p-1}+c\left(\|k\|_{L^{1}}\|x\|_{X}\right)^{p-1}\right] \\
\leq & \frac{3 T^{\beta}}{2 \Gamma(\beta+1)}\left[a+\left(b+c\|k\|_{L^{1}}^{p-1}\right)\|x\|_{X}^{p-1}\right]
\end{aligned}
$$

and by Lemma 10 , for any $x \in B_{r}$, we have

$$
\begin{aligned}
& \|A x(t)\| \\
& \leq \int_{0}^{T}|G(t, s)| \cdot\|F N x(s)\|^{q-1} d s \\
& \leq \int_{0}^{T}|G(t, s)| d s \\
& \quad \quad \times\left(\frac{3 T^{\beta}}{2 \Gamma(\beta+1)}\left[a+\left(b+c\|k\|_{L^{1}}^{p-1}\right)\|x\|_{X}^{p-1}\right]\right)^{q-1}
\end{aligned}
$$




$$
\begin{aligned}
& \leq \mu_{0} \\
& \cdot\left(\frac{3 T^{\beta}}{2 \Gamma(\beta+1)}\right. \\
& \left.\quad \times\left[\frac{r^{1 /(q-1)}}{2}\left(b+c\|k\|_{L^{1}}^{p-1}\right)+\left(b+c\|k\|_{L^{1}}^{p-1}\right) r^{p-1}\right]\right)^{q-1} \\
& \leq \mu_{0} \cdot\left(\frac{9 T^{\beta}\left(b+c\|k\|_{L^{1}}^{p-1}\right)}{4 \Gamma(\beta+1)}\right)^{q-1} r
\end{aligned}
$$

where $(p-1)(q-1)=1$, and the definition of $B_{r}$ yields that $a \leq(1 / 2)\left(b+c\|k\|_{L^{1}}^{p-1}\right) r^{1-q}$.

On the other hand, from $\left(\mathrm{H}_{2}\right), \| g(t, x(t)\|\leq\| g(t, x(t)-$ $g(t, \theta)\|+\| g(t, \theta)\|\leq L\| x \|_{X}+g_{0}$, where $g_{0}=\sup _{t \in[0, T]}\|g(t, \theta)\|$ and $\|\bar{g}(x)\| \leq\|\bar{g}(x)-\bar{g}(\theta)\|+\|\bar{g}(\theta)\| \leq M\|x\|_{X}+\|\bar{g}(\theta)\|$; then for $t \in[0, T]$, we have

$$
\begin{aligned}
\|B x(t)\| & \leq \int_{0}^{T}|G(t, s)|\|g(s, x(s))\| d s+\left|\frac{(2 t-T)}{2\left(\eta_{1}+\eta_{2}\right)}\right|\|\bar{g}(x)\| \\
& \leq \mu_{0} \cdot\left(L\|x\|_{X}+g_{0}\right)+\frac{T M}{2\left|\eta_{1}+\eta_{2}\right|}\|x\|_{X}+\frac{T\|\bar{g}(\theta)\|}{2\left|\eta_{1}+\eta_{2}\right|} \\
& \leq \mu_{0} \cdot\left(L r+\frac{L}{4} r\right)+\frac{T M}{2\left|\eta_{1}+\eta_{2}\right|} r+\frac{1}{2\left|\eta_{1}+\eta_{2}\right|} \frac{r}{4} \\
& \leq\left(\frac{5}{4} \mu_{0} L+\frac{4 T M+1}{8\left|\eta_{1}+\eta_{2}\right|}\right) r
\end{aligned}
$$

for all $x \in B_{r}$. By $\rho<1$, we can obtain that $A x+B y \in B_{r}$ for $x, y \in B_{r}$.

Next, we will prove that $A: X \rightarrow X$ is compact and continuous.

According to (42), it is easy to know that $\exists M_{0}>0$ such that $\|A x\|_{X} \leq M_{0}$ for all $x \in B_{r}$, which yields that $\mathscr{F}\left(B_{r}\right)$ is uniformly bounded in $X$. In view of the ArzeláAscoli theorem, we are going to prove that $\mathscr{F}\left(B_{r}\right) \subset X$ is equicontinuous.

For all $x \in B_{r}$, the inequality of (41) guarantees that $\|F N x(t)\| \leq\left(3 T^{\beta} / 2 \Gamma(\beta+1)\right)\left[a+\left(b+c\|k\|_{L^{1}}^{p^{-1}}\right) r^{p-1}\right]=: \widetilde{\mathscr{M}}$; then for $0 \leq \tau_{1}<\tau_{2} \leq T$, we have

$$
\begin{aligned}
& \left\|A x\left(\tau_{2}\right)-A x\left(\tau_{1}\right)\right\| \\
& =\| \int_{0}^{\tau_{2}} \frac{\left(\tau_{2}-s\right)^{\alpha-1}}{\Gamma(\alpha)} \phi_{q}(F N x(s)) d s \\
& \quad-\int_{0}^{\tau_{1}} \frac{\left(\tau_{1}-s\right)^{\alpha-1}}{\Gamma(\alpha)} \phi_{q}(F N x(s)) d s \| \\
& =\| \frac{1}{\Gamma(\alpha)} \int_{0}^{\tau_{1}}\left[\left(\tau_{2}-s\right)^{\alpha-1}-\left(\tau_{1}-s\right)^{\alpha-1}\right] \phi_{q}(F N x(s)) d s \\
& \quad+\frac{1}{\Gamma(\alpha)} \int_{\tau_{1}}^{\tau_{2}}\left(\tau_{2}-s\right)^{\alpha-1} \phi_{q}(F N x(s)) d s \|
\end{aligned}
$$

$$
\begin{gathered}
\leq \frac{\widetilde{\mathscr{M}}^{q-1}}{\Gamma(\alpha)}\left\{\int_{0}^{\tau_{1}}\left|\left(\tau_{2}-s\right)^{\alpha-1}-\left(\tau_{1}-s\right)^{\alpha-1}\right| d s\right. \\
\left.\quad+\int_{\tau_{1}}^{\tau_{2}}\left(\tau_{2}-s\right)^{\alpha-1} d s\right\} \\
=\frac{\widetilde{M}^{q-1}}{\Gamma(\alpha+1)}\left[2\left(\tau_{2}-t_{1}\right)^{\alpha}-\tau_{2}^{\alpha}+\tau_{1}^{\alpha}\right] .
\end{gathered}
$$

Since $t^{\alpha}$ is uniformly continuous on $[0, T]$, we can obtain that $T\left(B_{r}\right) \subset X$ is equicontinuous on $[0, T]$. Then, the ArzeláAscoli theorem yields that $T\left(B_{r}\right)$ is relatively compact in $X$.

Now, we show that $A: X \rightarrow X$ is continuous.

Let $\left\{x_{n}\right\} \subseteq X$ be a sequence with $x_{n} \rightarrow x$ in $X$; we will show that $\left\|A x_{n}-A x\right\|_{X} \rightarrow 0$. By the continuity of $f$, it is easy to see $\lim _{n \rightarrow \infty} N x_{n}(t)=N x(t)$. Moreover, using the Lebesgue dominated convergence theorem, we have

$$
\begin{aligned}
& \lim _{n \rightarrow \infty} F N x_{n}(t) \\
& =\lim _{n \rightarrow \infty}\left(\int_{0}^{t} \frac{(t-s)^{\beta-1}}{\Gamma(\beta)} N x_{n}(s) d s-\int_{0}^{T} \frac{(T-s)^{\beta-1}}{2 \Gamma(\beta)} N x_{n}(s) d s\right) \\
& =\int_{0}^{t} \frac{(t-s)^{\beta-1}}{\Gamma(\beta)} N x(s) d s-\int_{0}^{T} \frac{(T-s)^{\beta-1}}{2 \Gamma(\beta)} N x(s) d s \\
& =\operatorname{FN} x(t)
\end{aligned}
$$

uniformly for $t \in[0, T]$. Moreover, by the properties of Green function $G(t, s)$ and $\phi_{q}$, and the uniformly continuity of function $g$, we have

$$
\begin{aligned}
& \lim _{n \rightarrow \infty} A x_{n}(t) \\
& \quad=\lim _{n \rightarrow \infty}\left(\int_{0}^{T} G(t, s)\left[\phi_{q}\left(F N x_{n}(s)\right)-g\left(s, x_{n}(s)\right)\right] d s\right) \\
& \quad=\int_{0}^{T} G(t, s)\left[\phi_{q}(F N x(s))-g(s, x(s))\right] d s=A x(t)
\end{aligned}
$$

uniformly for $t \in[0, T]$. Thus we have $\left\|T x_{n}-T x\right\|_{X} \rightarrow 0$ in $X$. This shows that $A: X \rightarrow X$ is continuous.

Finally, we show that $B: X \rightarrow X$ is a contraction mapping. For $x, y \in E$, by $\left(\mathrm{H}_{2}\right)$, we obtain

$$
\begin{aligned}
& \sup _{t \in[0, T]}\|B x(t)-B y(t)\| \\
& =\sup _{t \in[0, T]}\left\|\int_{0}^{T} G(t, s)[g(s, x(s))-g(s, y(s))] d s\right\| \\
& \leq \sup _{t \in[0, T]} \int_{0}^{T}|G(t, s)| L\|x(s)-y(s)\| d s \\
& \leq \sup _{t \in[0, T]} L \int_{0}^{T}|G(t, s)| d s\|x-y\|_{X} \\
& \leq \mu_{0} L\|x-y\|_{X} .
\end{aligned}
$$

Thus $\|B x-B y\|_{X}<\rho\|x-y\|_{X}$, where $\rho<1$, which guarantees that $B: X \rightarrow X$ is a contraction mapping. 
Thus all the assumptions of Lemma 6 are satisfied and the conclusion of Lemma 6 implies that the operator $\mathscr{F}=A+B$ has at least a fixed point in $X$, which is a solution of BVP (1).

The proof is complete.

Obviously, if $\left(\mathrm{H}_{1}\right)-\left(\mathrm{H}_{3}\right)$ hold and

$$
\begin{aligned}
\rho & =\mu \cdot\left[\left(\frac{9 T^{\beta}\left(b+c\|k\|_{L^{1}}^{p-1}\right)}{4 \Gamma(\beta+1)}\right)^{q-1}+\frac{3 L}{2}+\frac{T\left\|x_{1}\right\|}{2\left|\eta_{1}+\eta_{2}\right|}\right] \\
& <1,
\end{aligned}
$$

with

$$
\mu=\frac{2 T^{\alpha}}{\Gamma(\alpha+1)}+\frac{T^{\alpha}}{2\left|\eta_{1}+\eta_{2}\right|}\left[\frac{|\lambda| T^{2}}{\Gamma(\alpha+2)}+\frac{|\lambda| T^{2}+2 \alpha\left|\eta_{2}\right|}{2 \Gamma(\alpha+1)}\right],
$$

then the following BVP:

$$
\begin{array}{r}
D_{0^{+}}^{\beta} \phi_{p}\left(D_{0^{+}}^{\alpha} x(t)+g(t, x(t))\right)=f(t, x(t), S x(t)), \\
t \in[0, T], \\
x(0)+x(T)=D_{0^{+}}^{\alpha} x(0)+D_{0^{+}}^{\alpha} x(T)=\theta, \\
\eta_{1} x^{\prime}(0)+\eta_{2} x^{\prime}(T)=\lambda \int_{0}^{T} x(t) d t+x_{1}, \\
\lambda \in \mathbb{R}, x_{1} \in E,
\end{array}
$$

has at least one solution in $X$.

In what follows, we will use the Schauder's fixed Point theorem to prove the existence of the solutions of BVP (1). We first list the following conditions.

$\left(\mathrm{A}_{1}\right)$ Let $f:[0, T] \times E^{2} \rightarrow E$ be continuous, and there exist $a_{i} \in \mathbb{R}^{+}(i=1,2,3)$ and $0 \leq l_{1}, l_{2}<p-1$ such that

$$
\begin{aligned}
&\|f(t, u, v)\| \leq a_{1}+a_{2}\|u\|^{l_{1}}+a_{3}\|v\|^{l_{2}}, \\
& \forall t \in[0, T], \quad u, v \in E .
\end{aligned}
$$

$\left(\mathrm{A}_{2}\right)$ The function $g:[0, T] \times E \rightarrow E$ is continuous and $g(0,-u)=-g(T, u)$ for all $u \in E$. Moreover, there exist $b_{1}, b_{2}>0$ and $0 \leq l_{3}<1$ such that

$$
\|g(t, u)\| \leq b_{1}+b_{2}\|u\|^{l_{3}} \quad \forall t \in[0, T], u, v \in E .
$$

$\left(\mathrm{A}_{3}\right)$ Let $\bar{g}: C(0, T ; E) \rightarrow E$ be a continuous functional, and there exist $b_{1}, b_{2}>0$ and $0 \leq l_{4}<1$ such that

$$
\|\bar{g}(x)\| \leq c_{1}+c_{2}\|x\|_{X}^{l_{4}} \quad \forall x \in X .
$$

Theorem 13. Assume that $\left(A_{1}\right)-\left(A_{3}\right)$ and $\left(H_{3}\right)$ hold. Then the $B V P(1)$ has at least one solution in $X$.

Proof. Define a bounded set $\Omega=\left\{x: x \in X,\|x\|_{X} \leq \mathscr{R}\right\}$, where

$$
\geq \max \left\{4 \mu_{0}\left(\frac{9 a_{1} T^{\beta}}{2 \Gamma(\beta+1)}\right)^{q-1},\left[\frac{9 a_{2} T^{\beta}\left(4 \mu_{0}\right)^{p-1}}{2 \Gamma(\beta+1)}\right]^{1 /\left(p-1-l_{1}\right)},\right.
$$

$$
\begin{gathered}
\left(4 \mu_{0} b_{2}\right)^{1 /\left(1-l_{3}\right)},\left[\frac{9 a_{3} T^{\beta}\left(4 \mu_{0}\right)^{p-1}\|k\|_{L^{1}}^{l_{2}}}{2 \Gamma(\beta+1)}\right]^{1 /\left(p-1-l_{2}\right)}, \\
\left.4\left(\mu_{0} b_{1}+\frac{T c_{1}}{2\left|\eta_{1}+\eta_{2}\right|}\right),\left(\frac{4 T c_{2}}{2\left|\eta_{1}+\eta_{2}\right|}\right)^{1 /\left(1-l_{4}\right)}\right\} .
\end{gathered}
$$

Now we are going to show that $\mathscr{F}: \Omega \rightarrow \Omega$. From the assumptions $\left(\mathrm{A}_{1}\right)$ and $\left(\mathrm{H}_{3}\right)$, we have

$$
\begin{aligned}
\|N x(t)\| & =\|f(t, x(t), S x(t))\| \\
& \leq a_{1}+a_{2}\|x(t)\|^{l_{1}}+a_{3}\|S x(t)\|^{l_{2}} \\
& \leq a_{1}+a_{2}\|x\|_{X}^{l_{1}}+a_{3}\left(\|k\|_{L^{1}}\|x\|_{X}\right)^{l_{2}}, \\
\Longrightarrow & \|F N x(t)\| \\
\leq & \int_{0}^{t} \frac{(t-s)^{\beta-1}}{\Gamma(\beta)}\|N x(s)\| d s \\
& +\int_{0}^{T} \frac{(T-s)^{\beta-1}}{2 \Gamma(\beta)}\|N x(s)\| d s \\
\leq & \frac{3 T^{\beta}}{2 \Gamma(\beta+1)}\left[a_{1}+a_{2}\|x\|_{X}^{l_{1}}+a_{3}\left(\|k\|_{L^{1}}\|x\|_{X}\right)^{l_{2}}\right] \\
\leq & \frac{3 a_{1} T^{\beta}}{2 \Gamma(\beta+1)}+\frac{3 a_{2} T^{\beta}}{2 \Gamma(\beta+1)}\|x\|_{X}^{l_{1}}+\frac{3 a_{3}\|k\|_{L^{1}}^{l_{2}}{ }^{\beta}}{2 \Gamma(\beta+1)}\|x\|_{X}^{l_{2}} \\
\leq & \frac{1}{3}\left(\frac{1}{4 \mu_{0}} \mathscr{R}\right)^{1 /(q-1)}+\frac{1}{3\left(4 \mu_{0}\right)^{p-1}} \mathscr{R}^{p-1-l_{1}} \mathscr{R}^{l_{1}} \\
& +\frac{1}{3\left(4 \mu_{0}\right)^{p-1}} \mathscr{R}^{p-1-l_{2}} \mathscr{R}^{l_{2}} \\
\leq & \left(\frac{1}{4 \mu_{0}} \mathscr{R}\right)^{p-1} \cdot
\end{aligned}
$$

By using of $\left(\mathrm{A}_{2}\right)$ and $\left(\mathrm{A}_{3}\right)$, we have

$$
\begin{aligned}
& \|\mathscr{F} x(t)\| \\
& \leq \| \int_{0}^{T} G(t, s)\left[\phi_{q}(F N x(s))-g(s, x(s))\right] d s \\
& \quad+\frac{(2 t-T)}{2\left(\eta_{1}+\eta_{2}\right)} \bar{g}(x) \| \\
& \leq \int_{0}^{T}|G(t, s)|\left(\left\|\phi_{q}(F N x(s))\right\|+\|g(s, x(s))\|\right) d s \\
& \quad+\left|\frac{(2 t-T)}{2\left(\eta_{1}+\eta_{2}\right)}\right|\|\bar{g}(x)\| \\
& \leq \int_{0}^{T}|G(t, s)| d s\left[\left(\left(\frac{1}{4 \mu_{0}} \mathscr{R}\right)^{p-1}\right)^{q-1}+b_{1}+b_{2}\|x\|_{X}^{l_{3}}\right] \\
& \quad+\frac{T}{2\left|\eta_{1}+\eta_{2}\right|}\left(c_{1}+c_{2}\|x\|_{X}^{l_{4}}\right) \\
& \leq \mu_{0}\left(\frac{1}{4 \mu_{0}} \mathscr{R}\right)+\mu_{0} b_{2}\|x\|_{X}^{l_{3}}+\frac{T c_{2}}{2\left|\eta_{1}+\eta_{2}\right|}\|x\|_{X}^{l_{4}}
\end{aligned}
$$




$$
\begin{aligned}
& +\mu_{0} b_{1}+\frac{T c_{1}}{2\left|\eta_{1}+\eta_{2}\right|} \\
\leq & \frac{\mathscr{R}}{4}+\frac{1}{4} \mathscr{R}^{1-l_{3}} \mathscr{R}^{l_{3}}+\frac{1}{4} \mathscr{R}^{1-l_{4}} \mathscr{R}^{l_{4}}+\frac{\mathscr{R}}{4}=\mathscr{R} .
\end{aligned}
$$

Hence, $\mathscr{F}(\Omega) \subseteq \Omega$. Similarly to the proof of Theorem 12 , it can be shown that the operator $\mathscr{F}: \Omega \rightarrow \Omega$ is completely continuous. According to the Schauder's fixed point theorem, $\mathscr{F}$ has at least a fixed point which is a solution of the problem (1).

The proof is completed.

\section{Example}

In this section, an example is given to illustrate our results.

Example 1. Consider the nonlocal boundary value problem for nonlinear fractional differential equation as follows

$$
\begin{gathered}
D_{0^{+}}^{2 / 3} \phi_{4}\left(D_{0^{+}}^{3 / 2} x(t)-\left(t-\frac{1}{2}\right)^{4} \sin x(t)\right) \\
=\frac{\arctan t}{10 e^{|x(t)|}}+\frac{x^{3}(t)}{100(1+|x(t)|)}+\left(\int_{0}^{1} \frac{\cos s}{8} x(s) d s\right)^{3}, \\
x(0)+x(1)=D_{0^{+}}^{3 / 2} u(0)+D_{0^{+}}^{3 / 2} u(1)=0, \\
x^{\prime}(0)+3 x^{\prime}(1)=2 \int_{0}^{1} x(t) d t+\frac{1}{10},
\end{gathered}
$$

where $t \in[0,1], T=1$. Then (58) has at least one solution.

Proof. It is easy to see that (58) is a form of (1) in the space $E=\mathbb{R}$. We have $p=4, q=4 / 3, \alpha=3 / 2, \beta=2 / 3, \eta_{1}=$ $1, \eta_{2}=3$ and

$$
\begin{gathered}
f(t, u, v)=\frac{\arctan t}{10 e^{|u|}}+\frac{u^{3}}{100(1+|u|)}+v^{3}, \\
g(t, u)=\left(t-\frac{1}{2}\right)^{4} \sin u, \\
S x(t)=\int_{0}^{t} h(s, x(s)) d s, \quad h(t, u)=\frac{\cos t}{8} u, \\
\bar{g}(x)=2 \int_{0}^{1} x(t) d t+\frac{1}{10} \quad \text { for } x \in C(0,1 ; \mathbb{R}) .
\end{gathered}
$$

Obviously, $f \in C\left([0,1] \times \mathbb{R}^{2}, \mathbb{R}\right), g \in C([0,1] \times \mathbb{R}, \mathbb{R})$, and $\bar{g} \in C(0,1 ; \mathbb{R}) \rightarrow \mathbb{R}$ is continuous functional. Moreover, $f, g, \bar{g}$ satisfy

$$
\begin{gathered}
|f(t, u, v)| \leq \frac{\pi}{20}+\frac{1}{100}|u|^{3}+|v|^{3} \quad \text { for } u, v \in \mathbb{R}, \\
\left|\bar{g}\left(x_{1}\right)-\bar{g}\left(x_{2}\right)\right| \leq 2|| x_{1}-x_{2} \|, \quad \text { for } x_{1}, x_{2} \in C(0,1 ; \mathbb{R}), \\
|g(t, u)-g(t, v)| \leq \frac{1}{16}|u-v|, \\
|h(t, u)| \leq \frac{\cos t}{8}|u| \quad \text { for } u, v \in \mathbb{R},
\end{gathered}
$$

and $g(0,-u)=-g(1, u)=-(1 / 16) \sin u$. Then, we get $a=$ $\pi / 20, b=1 / 100, c=1, M=2, L=1 / 16, k(t)=(1 / 8) \cos t$, and $\|k\|_{L^{1}}=(1 / 8) \sin 1\left(k \in L^{1}(0,1 ; \mathbb{R})\right)$ such that conditions $\left(\mathrm{H}_{1}\right)-\left(\mathrm{H}_{4}\right)$ hold. Moreover,

$$
\begin{aligned}
\mu_{0}= & \frac{\left|\eta_{2}\right| T^{\alpha}}{2\left|\eta_{1}+\eta_{2}\right| \Gamma(\alpha)}+\frac{3 T^{\alpha}}{2 \Gamma(\alpha+1)} \\
= & \frac{|3| \cdot 1^{3 / 2}}{2 \cdot|1+3| \cdot \Gamma(3 / 2)}+\frac{3 \cdot 1^{3 / 2}}{2 \cdot \Gamma(3 / 2+1)} \\
\approx & 1.5515 \\
\rho= & \mu_{0}\left(\frac{9 T^{\beta}\left(b+c\|k\|_{L^{1}}^{p-1}\right)}{4 \Gamma(\beta+1)}\right)^{q-1}+\frac{5}{4} \mu_{0} L+\frac{4 T M+1}{8\left|\eta_{1}+\eta_{2}\right|}<1 \\
= & \mu_{0} \cdot\left[\frac{9 \cdot 1^{2 / 3} \cdot\left(1 / 100+1 \times(\sin 1 / 8)^{4-1}\right)}{4 \times \Gamma(1 / 2+1)}\right]^{4 / 3-1} \\
& +\frac{5}{4} \cdot \mu_{0} \cdot \frac{1}{16}+\frac{4 \cdot 1 \cdot 2+1}{8 \cdot|1+4|} \\
\approx & 0.8755<1 .
\end{aligned}
$$

Hence, (58) satisfies all assumptions of Theorem 12. As a result, (58) has at least one solution.

\section{Conclusions}

In this paper, we study the existence solutions of nonlinear Caputo fractional integrodifferential equations with $p$ Laplacian operator and nonlocal boundary conditions in Banach spaces. We mainly consider the equivalent integral equations of corresponding fractional differential equations. By using via Krasnoselskii's fixed point theorem and Schauder's fixed point theorem, we obtain some new existence results for this kind of nonlocal boundary value problems (1). An illustrative example is also discussed to show the effectiveness of the results in this paper. In the near future, we will consider Riemann-Liouville Fractional nonlocal boundary problems, which will be more complicated.

\section{Acknowledgments}

This project is supported by NNSF of China Grants nos. 11271087, 61263006, Guangxi Scientific Experimental (ChinaASEAN Research) Centre no. 20120116, the open fund of Guangxi Key laboratory of Hybrid Computation and IC Design Analysis no. 2012HCIC07, and the Innovation Project of Guangxi University for Nationalities no. gxunchx2012096.

\section{References}

[1] G. Q. Chai, "Positive solutions for boundary value problem of fractional differential equation with p-Laplacian operator," Boundary Value Problems, p. 2012, article 18, 2012. 
[2] T. Y. Chen, W. Liu, and Z. G. Hu, "A boundary value problem for fractional differential equation with $p$-Laplacian operator at resonance," Nonlinear Analysis. Theory, Methods \& Applications, vol. 75, no. 6, pp. 3210-3217, 2012.

[3] T. Y. Chen and W. B. Liu, "An anti-periodic boundary value problem for the fractional differential equation with a $p$ Laplacian operator," Applied Mathematics Letters, vol. 25, no. 11, pp. 1671-1675, 2012.

[4] A. A. Kilbsa, H. M. Srivastava, and J. J. Trujillo, Theory and Applications of Fractional Differential Equations, Elsevier, Amsterdam, The Netherlands, 2006.

[5] X. Y. Liu and Z. H. Liu, "Existence results for fractional differential inclusions with multivalued term depending on lower-order derivative," Abstract and Applied Analysis, vol. 2012, Article ID 423796, 24 pages, 2012.

[6] Z. H. Liu and X. W. Li, "On the controllability of impulsive fractional evolution inclusions in Banach spaces," Journal of Optimization Theory and Applications, vol. 156, no. 1, pp. 167$182,2013$.

[7] Z. H. Liu and D. Motreanu, "A class of variational-hemivariational inequalities of elliptic type," Nonlinearity, vol. 23, no. 7, pp. 1741-1752, 2010.

[8] Z. H. Liu and J. H. Sun, "Nonlinear boundary value problems of fractional differential systems," Computers \& Mathematics with Applications, vol. 64, no. 4, pp. 463-475, 2012.

[9] Z. H. Liu and J. H. Sun, "Nonlinear boundary value problems of fractional functional integro-differential equations," Computers \& Mathematics with Applications, vol. 64, no. 10, pp. 3228-3234, 2012.

[10] I. Podlubny, Fractional Differential Equations, Academic Press, San Diego, Calif, USA, 1999.

[11] F. Wang, Z.-H. Liu, and J. Li, "Complete controllability of fractional neutral differential systems in abstract space," Abstract and Applied Analysis, vol. 2013, Article ID 529025, 11 pages, 2013.

[12] H. Okochi, "On the existence of periodic solutions to nonlinear abstract parabolic equations," Journal of the Mathematical Society of Japan, vol. 40, no. 3, pp. 541-553, 1988.

[13] A. Alsaedi, "Existence of solutions for integrodifferential equations of fractional order with antiperiodic boundary conditions," International Journal of Differential Equations, vol. 2009, Article ID 417606, 9 pages, 2009.

[14] J. B. Liu and Z. H. Liu, "On the existence of anti-periodic solutions for implicit differential equations," Acta Mathematica Hungarica, vol. 132, no. 3, pp. 294-305, 2011.

[15] Z. H. Liu, "Anti-periodic solutions to nonlinear evolution equations," Journal of Functional Analysis, vol. 258, no. 6, pp. 2026-2033, 2010.

[16] E. Kaslik and S. Sivasundaram, "Non-existence of periodic solutions in fractional-order dynamical systems and a remarkable difference between integer and fractional-order derivatives of periodic functions," Nonlinear Analysis. Real World Applications, vol. 13, no. 3, pp. 1489-1497, 2012.

[17] M. S. Tavazoei and M. Haeri, "A proof for non existence of periodic solutions in time invariant fractional order systems," Automatica, vol. 45, no. 8, pp. 1886-1890, 2009.

[18] M. S. Tavazoei, "A note on fractional-order derivatives of periodic functions," Automatica, vol. 46, no. 5, pp. 945-948, 2010.

[19] M. S. Tavazoei and M. Haeri, "Simplification in the proof presented for nonexistence of periodic solutions in time invariant fractional order systems," In press, http://arxiv.org/abs/1202 .5878 .
[20] S. C. Lim, M. Li, and L. P. Teo, "Langevin equation with two fractional orders," Physics Letters A, vol. 372, no. 42, pp. 63096320, 2008.

[21] S. C. Lim and L. P. Teo, "The fractional oscillator process with two indices," Journal of Physics A, vol. 42, no. 6, Article ID 065208, 34 pages, 2009.

[22] B. Ahmad and J. J. Nieto, "Sequential fractional differential equations with three-point boundary conditions," Computers \& Mathematics with Applications, vol. 64, no. 10, pp. 3046-3052, 2012.

[23] B. Ahmad, J. J. Nieto, and A. Alsaedi, "A nonlocal three-point inclusion problem of Langevin equation with two different fractional orders," Advances in Difference Equations, vol. 2012, article 54, 2012.

[24] B. Ahmad, J. J. Nieto, A. Alsaedi, and M. El-Shahed, "A study of nonlinear Langevin equation involving two fractional orders in different intervals," Nonlinear Analysis. Real World Applications, vol. 13, no. 2, pp. 599-606, 2012.

[25] B. Ahmad and J. J. Nieto, "Solvability of nonlinear Langevin equation involving two fractional orders with Dirichlet boundary conditions," International Journal of Differential Equations, vol. 2010, Article ID 649486, 10 pages, 2010.

[26] A. P. Chen and Y. Chen, "Existence of solutions to nonlinear Langevin equation involving two fractional orders with boundary value conditions," Boundary Value Problems, vol. 2011, Article ID 516481, 17 pages, 2011.

[27] D. R. Smart, Fixed Point Theorems, Cambridge University Press, London, UK, 1980. 


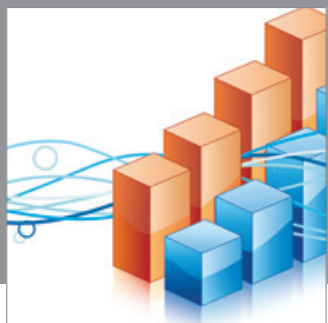

Advances in

Operations Research

mansans

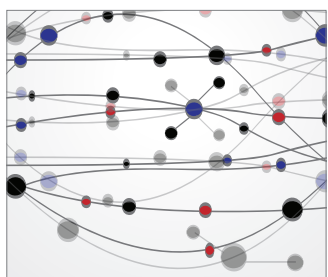

The Scientific World Journal
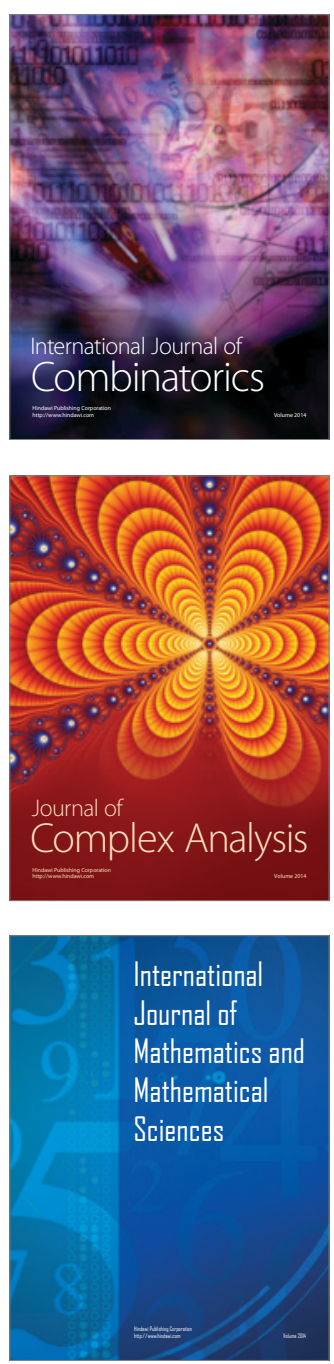
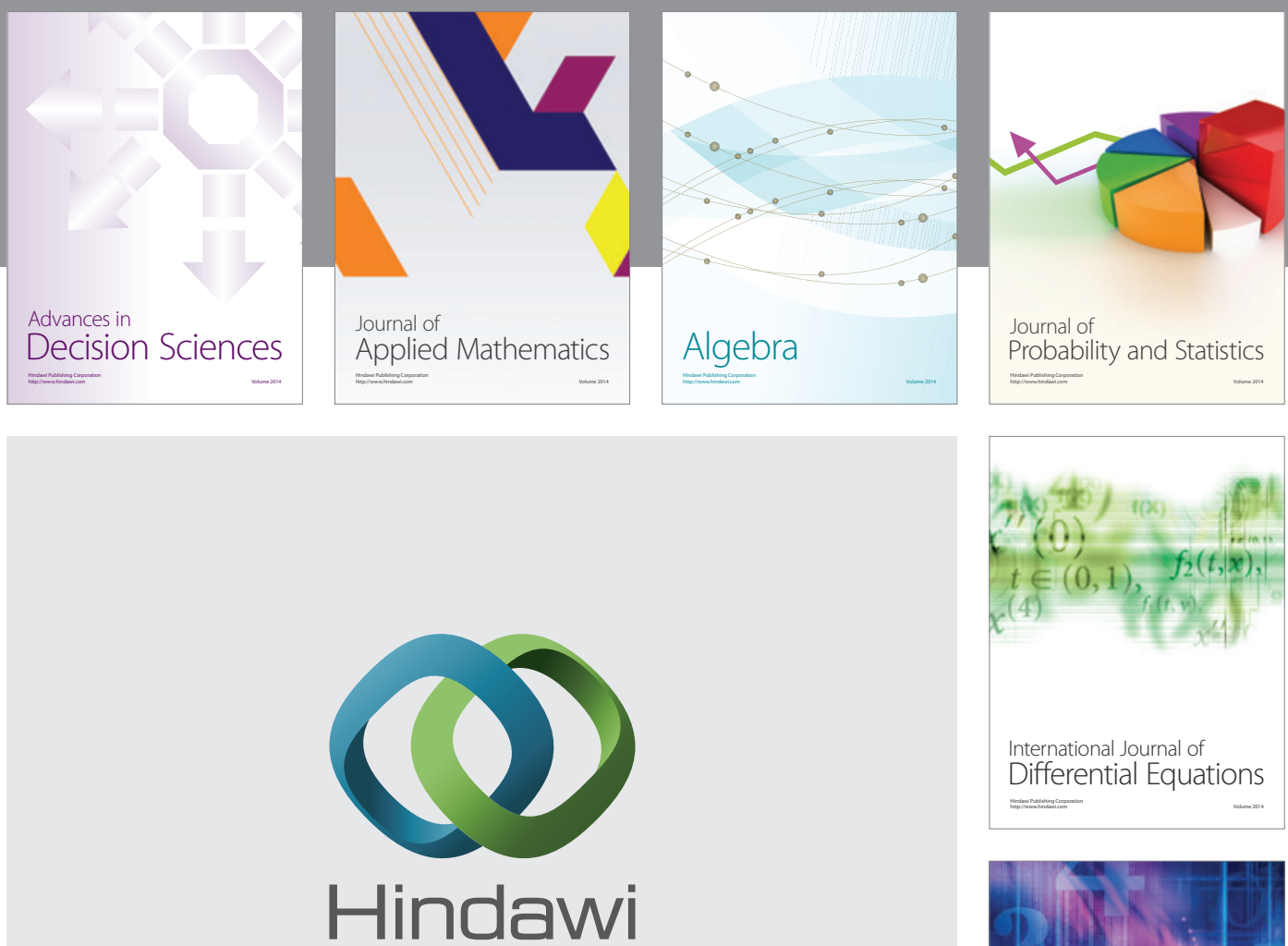

Submit your manuscripts at http://www.hindawi.com
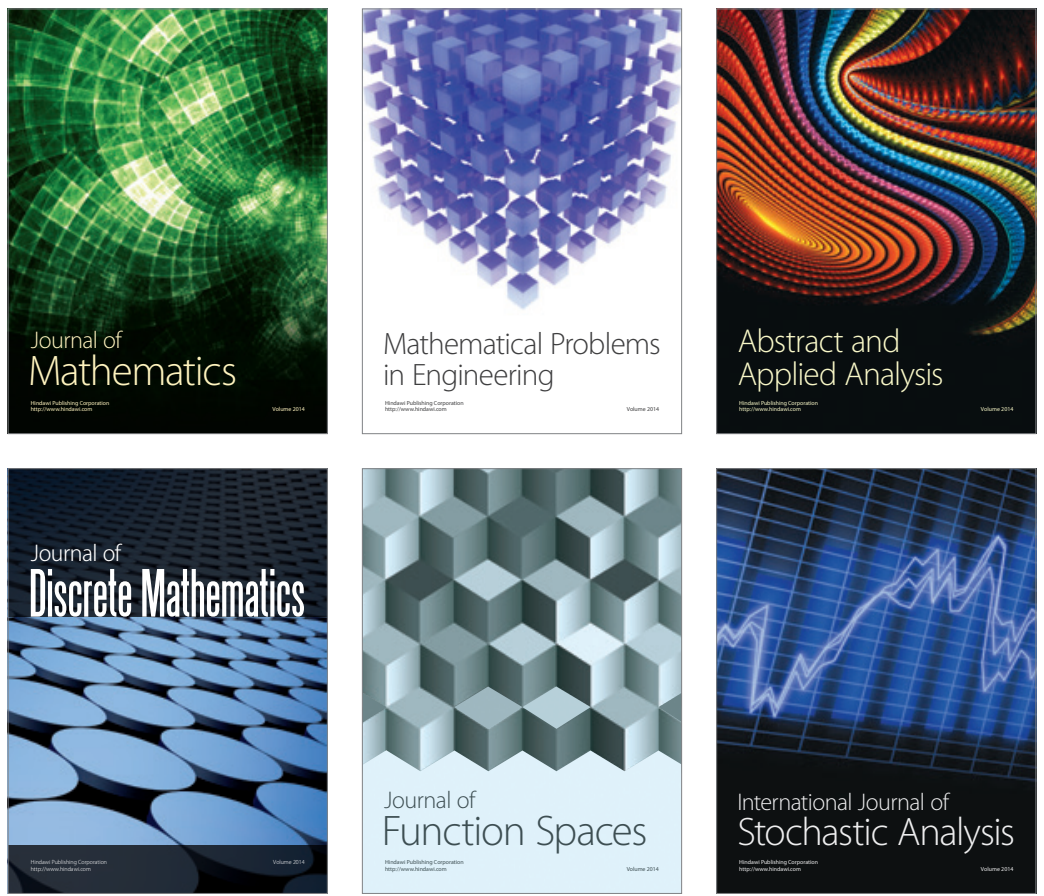

Journal of

Function Spaces

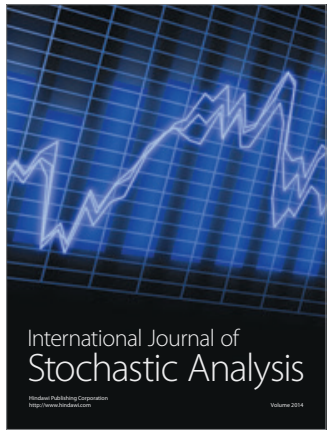

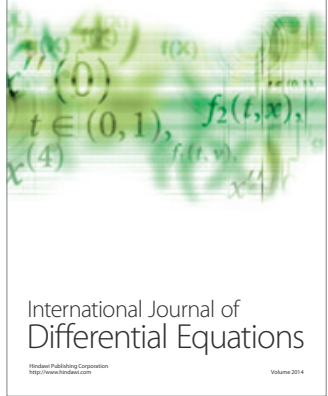
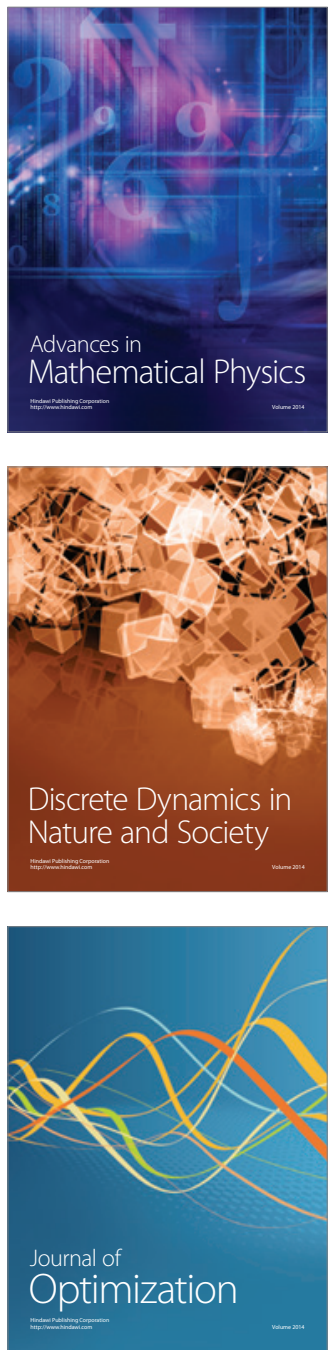